\title{
Investigation of die drool phenomenon for HDPE polymer melt
}

\author{
Jan Musil, Martin Zatloukal *
}

\begin{abstract}
A B S T R A C T
I $n$ this work, the die drool phenomenon analysis has been performed for HDPE polymer melt by using a specific type of experimental set-up, digital image analysis technique and rheological tools. It has been revealed that the thermally induced degradation occurring inside the processing equipment may lead to HDPE melt elasticity enhancement, which promotes unwanted material accumulation at the end of the extrusion die.
\end{abstract}

\section{Introduction}

Due to the viscoelastic nature of the polymer melts, unwanted flow phenomena such as die swell, shark skin, die drool, interfacial instabilities, etc. (Bird et al., 1987; Tanner, 1985; Hatzildriakos and Migler, 2005; Klein, 1981; Kurtz and Szaniszlo, 1991; Hogan et al., 2009; Holtzen and Musiano, 1996; Priester, 1994; Gander and Giacomin, 1997; Ding et al., 2000; Dhori et al., 1997; Chaloupkova and Zatloukal, 2007, 2009; Pivokonsky et al, 2008; Zatloukal et al, 2002, 2005, 2005, 2004, 2002, 2001; Martyn et al., 2004, 2009; Zatloukal and Witte, 2006; Shelby and Caflisch, 2004) can occur. The stabilization of these flow phenomena during polymer processing is usually difficult because the relationship between the polymer rheology, equipment design, processing conditions and the flow instabilities is not fully understood yet.

Die drool, also called die build-up, die bleed or plate-out, is the undesirable spontaneous accumulation of polymer melt at the exit edges or open faces of extrusion die during melt extrusion of polyolefins, PVC or filled polymers. This unwanted phenomenon can occur in all common extrusion processes (film blowing, pipe or profile extrusion, fiber spinning, film casting, etc.) and after it arises, the productivity of extrusion line rapidly decreases for the following basic reasons. Firstly, a small amount of accumulated and usually degradated material sticks to the extrudate at the die exit region, which causes depreciation of the final product. In the case of film casting or extrusion of solid profiles it is possible to solve this problem only by stripping of the accumulated material from the die exit region. However, in the case of film blowing, pipe, cored profile extrusion or wire sheathing the die drool arises not only at the outer exit region but also at the inner one, thereby the first clean opportunity is impossible. Therefore, in these cases, the extrusion line is necessary to periodically switch off to clean the die, which is uneconomical and time consuming.

The fundamental problem of die drool phenomenon is to understand and describe the formation principle of this phenomenon. In the open literature, several factors have been found to support the formation of this instability. They include pressure fluctuations in screw (Klein, 1981), volatiles, low molecular fractions of the polymer, fillers, poor dispersion of pigments (Kurtz and Szaniszlo, 1991), die swell (Klein, 1981; Hogan et al., 2009), processing near degradation temperature (Holtzen and Musiano, 1996), dissimilar viscosities in blends (Priester, 1994), die design (Gander and Giacomin, 1997; Ding et al., 2000; Dhori et al., 1997), etc.

Currently, two basic types of die drool phenomenon are known; external and internal. It has been recently found that the external type of die drool phenomenon for metallocene type of LLDPE polymer is predominantly driven by the extrudate free surface rupture and suction effect due to the negative pressure generated at the die exit region (Chaloupkova and Zatloukal, 2007, 2009), which can be suppressed by the die exit angle modification (Chaloupkova and Zatloukal, 2007, 2009). On the other hand, the internal type of die drool phenomenon can be caused by the flow induced fractionation that occurred before the polymer melt leaves the extrusion die, which is not fully understood yet (Shelby and Caflisch, 2004). Thus, the effect of processing conditions on this unwanted flow phenomenon has been investigated in this work for unfiled HDPE extrusion through annular extrusion die by using a specific type of digital image analysis technique. 


\section{Material characterization}

In this work, HDPE Tipelin FS 450-26 (TVK, Hungary) polymer, widely used in film extrusion, was chosen for the experimental research. The basic material characteristics are provided in Table 1 (Pivokonsky et al., 2008). In order to evaluate the HDPE polymer

Table 1

\begin{tabular}{|c|c|}
\hline Property & HDPE Jípelin F5 450-26 \\
\hline Deusity (gcm $\left.{ }^{x}\right)$ & (1.545 \\
\hline$M_{w}\left(g i n u l^{-1}\right)$ & 212,300 \\
\hline $\mathrm{W}_{n}\left(\mathrm{~g} \mathrm{~mol}^{-1} \mathrm{j}\right.$ & 22,430 \\
\hline $\mathrm{WI}_{2}\left(\mathrm{gmol}^{\prime}\right)$ & $1,045,000$ \\
\hline$M_{4,} / M_{\mathrm{n}}$ (dimensionless) & 9.465 \\
\hline DSC peak meiting porint ( c) & 130.3 \\
\hline DSC heal of fusion $\left(\mathrm{Jg}^{-1}\right)$ & 190.3 \\
\hline Etystallinity $[\%]$ & 64.9 \\
\hline
\end{tabular}

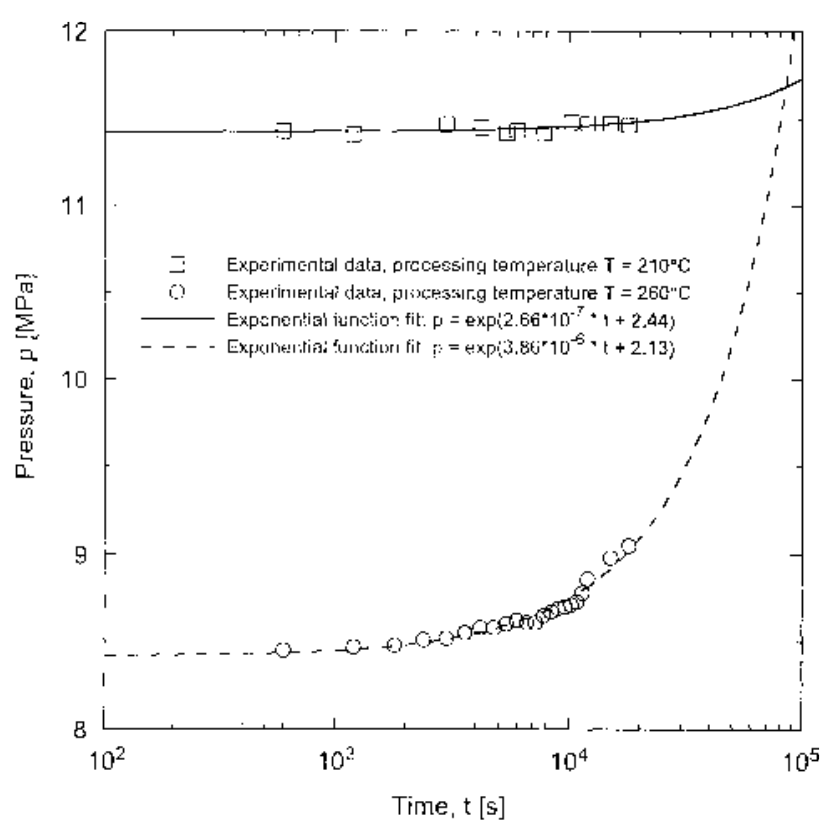

Fig. 1. Effect of processing temperature on the time dependent extrusion pressure for tested HDPE Tipelin FS 450-26 sample. thermal stability, firstly, polymer pellets were added and melted in the Rosand RH7-2 control speed capillary rheometer equipped by a capillary die having $16 \mathrm{~mm}$ length and $1 \mathrm{~mm}$ radius. After each $10 \mathrm{~min}$ of rest, the constant shear rate $\left(80 \mathrm{~s}^{-1}\right)$ has been applied and the equilibrium capillary pressure drop has been measured. This procedure was repeated 30 times to cover $5 \mathrm{~h}$ degradation time period after which the extruded polymer has been collected. This procedure was applied for two different melt temperatures $T=210$ and $260{ }^{\circ} \mathrm{C}$. Effect of processing temperature and time on the extrusion pressure for the tested HDPE sample is provided in Fig. 1. It is clearly visible that the extrusion pressure rise occurs during the time suggesting that temperature induced degradation leads to molecular weight increase of the tested polymer and the effect is much more pronounced for the higher temperature. In order to understand sample degradation in more detail, frequency dependent complex viscosity/recoverable shear and transient uniaxial extensional viscosity were determined for not degraded as well as degraded HDPE samples $\left(5 \mathrm{~h}\right.$ at $\left.210{ }^{\circ} \mathrm{C} / 260^{\circ} \mathrm{C}\right)$ by using the rotational rheometer from Advanced Rheometric Expansion System (ARES 2000, Rheometrics Scientific, USA) in parallel-plate mode and the Sentmanat Extensional Rheometer (SER-HV-A01, Xpansion Instruments, USA (Sentmanat et al., 2005, 2004; Sentmanat, 2003), see Fig. 2), respectively. These rheological characteristics are shown in Figs. 3-5. Based on the rheological data depicted in these figures, it can be concluded that thermally induced degradation of HDPE sample leads to increase of complex viscosity/shear elasticity (recoverable shear)/ uniaxial extensional viscosity and the effect is much more pronounced for the highest processing temperature $\left(\mathrm{T}=260^{\circ} \mathrm{C}\right)$.

It should be mentioned that the recoverable shear, $S r$, is the measure of the shear elasticity, which is given by the following equation:

$S \mathrm{r}=\frac{N_{1}}{2 \tau_{\gamma}}$

where $N-i$ is the first normal stress difference and $x_{x y}$ is the shear stress. Considering the Cox-Merz rule (Cox and Merz, 1958) and the similarity between $N^{\wedge} 2$ and storage modulus $G$ (Bird et al., 1987), Eq. (1) can be rewritten into the following form:

$S_{r}=\frac{G}{\eta \omega}$

where $r f$ is the complex viscosity and co is the frequency. In this work, the recoverable shear shown in Fig. 4 was calculated by using rotational rheometry data according to Eq. (2). a

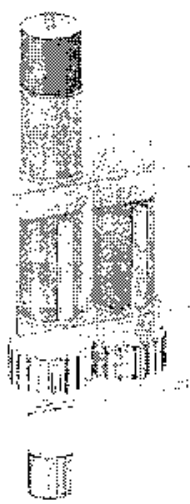

b

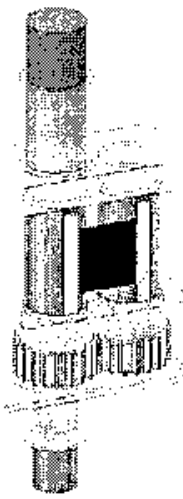

$\mathrm{C}$

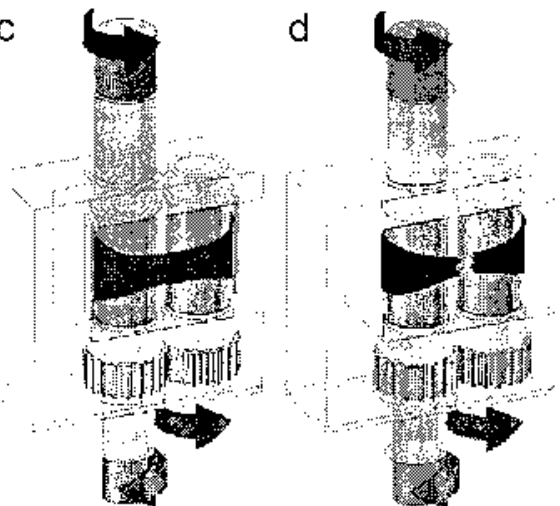

Fig. 2. Visualization methodology for the extensional viscosity measurements of the HDPE polymer melt by using a Sentmanat Extensional Rheometer (SER): (a) SER unit, (b) deposition of the polymer sample on the SER unit, (c), sample stretching and (d) sample at break. 